\title{
Predicting Obstetric Vasculopathies through Study of Diastolic Notch and other Indices of Resistance to Blood Flow in Uterine Artery
}

\author{
Pankaj Desai
}

\begin{abstract}
Obstetric vasculopathies are a set of apparently heterogeneous and poorly understood conditions. Conditions like recurrent miscarriages due to fetal demise, pre-eclampsia, IUGR, recurrent still births and accidental hemorrhage all seem to have the same underlying etiopathology. They are grouped into one large set coined as 'Obstetric Vasculopathies'. Resistance to blood flow in the uterine arteries can be important and effective method to predict obstetric vasculopathies (except recurrent miscarriages). Resistance to this blood flow can be measured by the presence (and subsequent disappearance) of diastolic notch as well as the standard color Doppler indices namely Pulsatility Index (PI), Resistance Index (RI) and Systolic: Diastolic (S: D) ratio. In normal pregnancy almost always at 12 to 14 weeks of duration, the uterine artery shows presence of a diastolic notch. In high-risk subjects, disappearance of diastolic notch at midtrimester in uterine artery Doppler waveform analysis if used alone may not be a good screening method for obstetric vasculopathies. However, the combination of a diastolic notch and an abnormal resistance index in both uterine arteries at 20 weeks gestation is more accurate indicator in predicting severe pregnancy complications. These subjects are almost eight times more likely to develop either: Clinically significant hypertension and/or deliver prior to 32 weeks and/or have a perinatal demise and/or have an Infant with a birth weight of less than $1500 \mathrm{gm}$. Bilateral or Unilateral: Studying bilateral uterine artery seems to be more accurate that unilateral artery for prediction of obstetric vasculopathies. Prediction in I-Trimester: Disappearance of uterine artery diastolic notch in combination with other parameters seems to be a good test for prediction of obstetric vasculopathies particularly PIH and IUGR in I-Trimester. The advantage of such an early allows an early instituting of preventive measures. Notch Depth Index (NDI): It is calculated as the depth of the diastolic notch divided by the maximal diastolic velocity. The NDI value in the second trimester is associated with the later onset of pre-eclampsia, and is clinically more useful in predicting pre-eclampsia than the two conventional indices. Abnormal maternal uterine artery Doppler in association with elevated maternal serum AFP, HCG, Inhibin A or decreased PAPP-A identifies a group of subjects at greater risk of IUGR and gestational hypertension with proteinuria. Lowdose aspirin administered as early as 14 to 16 weeks of gestation to pregnant subjects at high risk of pre-eclampsia with abnormal uterine Doppler findings may reduce or modify the course of severe pre-eclampsia and may help in prevention of IUGR.
\end{abstract}

Keywords: Color Doppler, Pre-eclampsia, IUGR, Vasculopathies, Diastolic notch, Uterine artery.

How to cite this article: Desai P. Predicting Obstetric Vasculopathies through Study of Diastolic Notch and other

Date of Received: 09-02-13

Date of Acceptance: 19-04-13

Date of Publication: January 2013
Indices of Resistance to Blood Flow in Uterine Artery. Int J Infertility Fetal Med 2013;4(1):24-30.

Source of support: Nil

Conflict of interest: None

\section{INTRODUCTION}

Normal pregnancy and normal delivery are two classic examples of diagnoses in retrospect. Only after a pregnancy ends uneventfully can one say that this one was 'normal'. An obstetrician in clinical practice is therefore always seeking for methods to predict conditions that can disturb the normalcy of an ongoing pregnancy. This can enable him/ her to take steps to prevent or minimize the complications.

\section{What are Obstetric Vasculopathies?}

Obstetric vasculopathies are a set of apparently heterogeneous and poorly understood conditions. They constitute the single largest group of derangements that can endanger the life of both-the mother and/or the unborn child. Seemingly unrelated obstetric diagnoses like recurrent miscarriages due to fetal demise, pre-eclampsia, IUGR, recurrent still births and accidental hemorrhage all seem to have the same underlying etiopathology. They are for that reason grouped into one large set coined as 'obstetric vasculopathies'.

There are many types of tests to predict obstetric vasculopathies. These can be largely grouped as clinical, biochemical and imaging. Many assorted tests have been found to have some role in predicting obstetric vasculopathies. Of these, imaging technology has been found to have maximum promise. Color Doppler as applied to obstetric conditions has been proved to be a game-changer in prediction, management decisions and prognostication. Resistance to blood flow in the uterine arteries can be important and effective method to predict obstetric vasculopathies. Resistance to this blood flow can be measured by the presence (and subsequent disappearance) of diastolic notch as well as the standard color Doppler indices namely pulsatility index (PI), resistance index (RI) and systolic: diastolic (S: D) ratio.

At this stage it need to be clarified that recurrent late miscarriages (occurring in late first trimester or early second trimester)-one important feature of obstetric vasculopathies 
is not focused here. These occur too early and are therefore not expected to be influenced by the blood flow in uterine arteries. However, the basic etiopathology of obstetric vasculopathies that occurs at fetomaternal interface remains applicable to all conditions bunched under obstetric vasculopathies including recurrent miscarriages. Nevertheless these miscarriages occur too early for the uterine artery changes to help in their prediction.

\section{WHAT IS DIASTOLIC NOTCH?}

In normal pregnancy almost always at 12 to 14 weeks of duration, the uterine artery shows presence of a diastolic notch (Fig. 1). This is the small dip found just before the diastolic wave begins. Presence of diastolic notch suggests that the second wave of trophoblastic invasion is still not complete. It also suggests that the process may be ongoing but still not over. Presence of diastolic notch in these subjects at 12 to 14 weeks may not have much significance except indicating that the process is ongoing. Disappearance of the diastolic notch seems to be a very reliable predictor of obstetric vasculopathy. This occurs at around mid-trimester or thereafter anytime.

\section{DIASTOLIC NOTCH AS A PREDICTOR OF OBSTETRIC VASCULOPATHIES}

Over a period of time disappearance of diastolic notch seems to be a popular predictor of obstetric vasculopathies especially pre-eclampsia and IUGR. Chan et al showed that the combination of a diastolic notch and an abnormal resistance index in both uterine arteries at 20 weeks gestation is the most accurate indicator in predicting severe pregnancy complications. These subjects are almost eight times more likely to develop either: Clinically significant hypertension and/or deliver prior to 32 weeks and/or have a perinatal demise and/or have an infant with a birth weight of less than 1500 gm. ${ }^{1}$

In one study, accuracy of various tests used to predict the onset of pre-eclampsia and the effectiveness of preventative treatment were reviewed. It was found that in general, tests in early pregnancy for predicting later development of pre-eclampsia have better specificity than sensitivity, as body mass index greater than 34, alphafetoprotein; fibronectin and uterine artery Doppler (bilateral notching) all have specificities above 90\%. Only uterine artery Doppler resistance index and combinations of indices have a sensitivity of over $60 \%{ }^{2}$

Gestational age of screening between 20 and 24 weeks has been recommended as the finest time for screening of high-risk pregnancies for obstetric vasculopathies. In one study it has been suggested that any notch (unilateral or bilateral) has a sensitivity of $76 \%$ and specificity of $95 \%$ for pre-eclampsia at this duration of pregnancy. For preeclampsia that requires delivery prior to 34 weeks, the sensitivity of persistence of diastolic notch at 20 to 24 weeks is over $90 \%$. Screening at 20 weeks has a higher sensitivity than screening at 24 weeks (81 vs 76\%), but lower specificity (87 vs 95\%). ${ }^{3}$

One study studied the longitudinal variation in uterine artery blood flow pattern in relation to birth weight. In this study the relationship between the timing of disappearance of high-resistance uterine artery waveforms between the first and second trimester of pregnancy and birth weight was focused upon. Four hundred eleven pregnancies showed absent or unilateral uterine artery notches at the 11 to 14-week scan (group 1). All these cases maintained a lowresistance uterine blood flow pattern at the second-trimester scan. Of the 251 pregnancies with bilateral notches at the 11 to 14-week scan, 222 subsequently displayed a lowresistance blood flow at 18 to 23 weeks (group 2) whereas only 29 maintained bilateral notches (group 3). The mean birth weight was higher in group 1 (3452 gm) than in groups 2 (3310 gm) and 3 (3224 gm). This difference was confirmed after adjusting for confounding variables. Small-forgestational-age fetuses were more common in groups 2 (14.9\%) and 3 (14\%) compared with group 1 (6.8\%, $\mathrm{p}<0.001$ ). During the study period, severe preterm preeclampsia was observed in 6 cases only. This study showed that the longitudinal variation in uterine artery blood flow pattern has a statistically significant correlation with birth weight, likely reflecting the timing and degree of trophoblastic invasion of the maternal vessels. ${ }^{4}$

Disappearance of diastolic notch at mid-trimester (Fig. 2) alone has also been studied for this purpose. However, one study has shown that in high-risk subjects, disappearance of diastolic notch at mid-trimester in uterine artery Doppler waveform analysis if used alone may not be a good screening method for obstetric vasculopathies. ${ }^{5}$

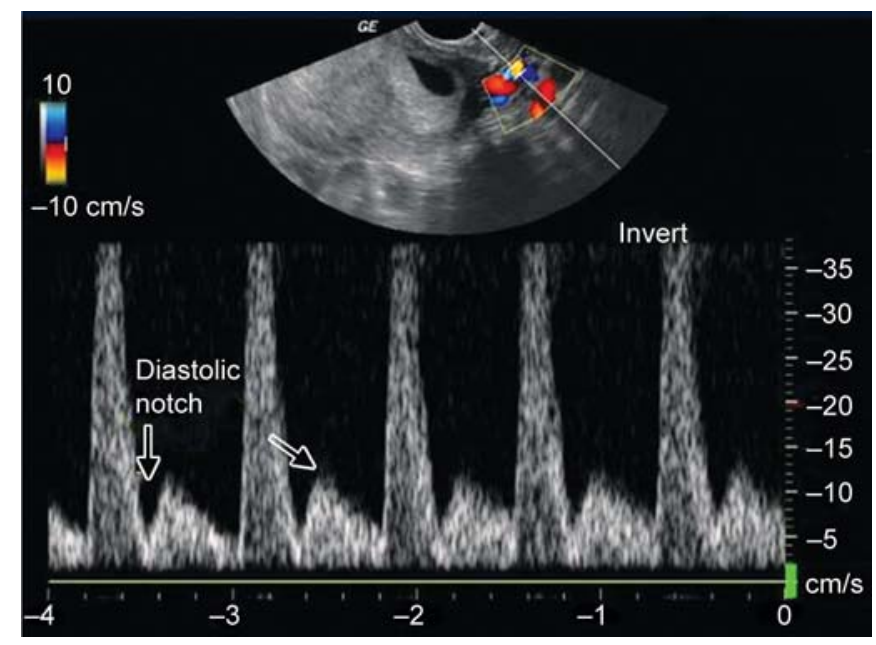

Fig. 1: Uterine artery diastolic notch 


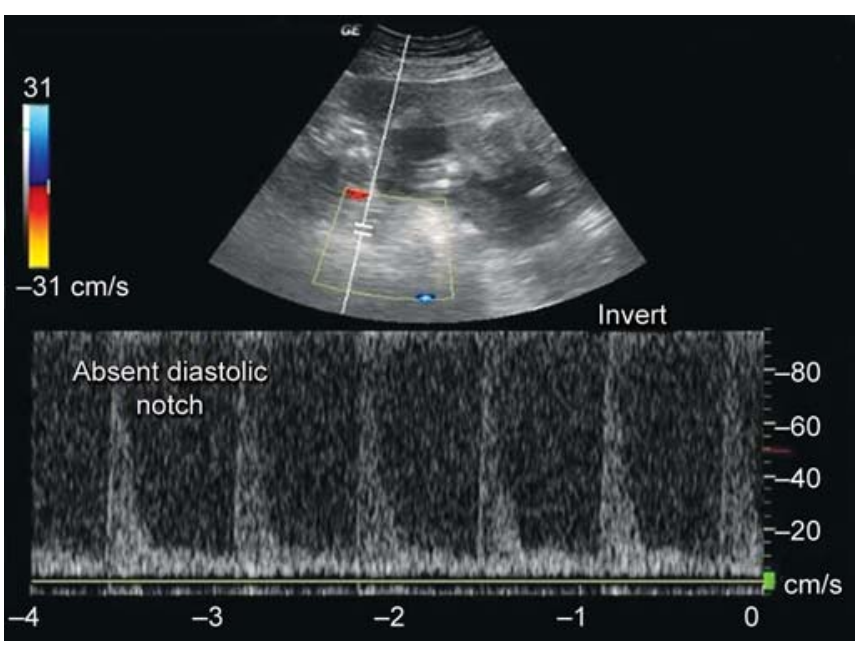

Fig. 2: Disappeared diastolic notch in second trimester

\section{Bilateral or Unilateral}

This matter seems to be interesting. In one study an analysis of the value of bilateral uterine artery notching in the second trimester in the risk assessment for pre-eclampsia, gestational hypertension, and small-for-gestational-age (SGA) without pre-eclampsia was studied. This prospective cohort study included 4190 singleton pregnancies that underwent ultrasound examination between 23 and 25 weeks' gestation. The 95th percentiles of the mean pulsatility index (PI) and resistive index (RI) of both uterine arteries were calculated. It was found that bilateral uterine notching between 23 and 25 weeks' gestation is an independent risk factor for the development of early-onset pre-eclampsia and gestational hypertension. The authors therefore suggested that bilateral uterine artery notching should be considered in the assessment of risk for the development of these pregnancy complications. ${ }^{6}$ In one review cited above too bilateral uterine artery notching seems to have been studied and found to be more competent than other methods for predicting obstetric vasculopathies. ${ }^{2}$

In another study reference range and characteristics of uterine artery Doppler in pregnant Thai subjects at 11 to 13 (+6) gestational weeks was studied. A 150 normal pregnant subjects at 11 to $13(+6)$ weeks of gestation who were attending at the antenatal care clinic were recruited. It was found that 39 patients (26\%) demonstrated unilateral notch, $12(8 \%)$ of the patients demonstrated ipsilateral notch, 19 patients (12.7\%) demonstrated contralateral notch, and eight (5.3\%) demonstrated unilateral notch with central placenta. ${ }^{8}$

In an attempt to construct reference ranges of uterine artery Doppler parameters against gestation in twin pregnancies and to present charts of the PI and RI for clinical use, 557 subjects with dichorionic twin pregnancies at 17 to 38 weeks' gestation were studied. Uterine artery measurements were performed by color and pulsed Doppler imaging. The uterine artery PI and RI were calculated as a mean of both sides. It was found that bilateral uterine artery Doppler indices in twin pregnancies are lower than in singleton pregnancies. ${ }^{9}$

One study was performed to evaluate uterine artery Doppler velocimetry (UADV) measurement of unilateral or bilateral abnormalities as a predictor of complications in pregnancy during the mid-second trimester (20-24 weeks). 1,090 pregnant subjects who had undergone UADV twice: Once between the 20th and 24th week (1st stage) and again between the 28th and 32nd week (2nd stage) of pregnancy were enrolled. UADV was performed bilaterally. Followup UADV was performed between the 28th and 32nd week, and the frequencies of pregnancy-induced hypertension (PIH), fetal growth restriction (FGR), and preterm delivery (before 34 weeks of gestation) were determined. Abnormal results found by UADV performed between the 20 and 24 weeks of pregnancy, such as high S/D ratios regardless of placental location and the presence of an early diastolic notch, were associated with significant increases in the incidences of intrauterine growth restriction (IUGR) and PIH. This was true for both bilateral and unilateral abnormalities. The authors concluded that abnormal findings in bilateral UADV during the second trimester especially warrant close follow-up for the detection of subsequent development of pregnancy complications. ${ }^{7}$

It is therefore seems that studying bilateral uterine artery seems to be more accurate that unilateral artery for prediction of obstetric vasculopathies.

\section{In First Trimester or Second Trimester?}

Disappearance of diastolic notch in mid-trimester seems to be a norm singly or in combinations with other parameters for prediction of obstetric vasculopathies especially PIH, and IUGR. Thus, second trimester seems to be the preferred period for this parameter. On the other hand there are studies which interestingly use diastolic notch in combination with other parameters in this prediction in first trimester rather successfully.

In clinical practice one sees many occasions when the diastolic notch may disappear in first trimester itself (Fig. 3). How accurate was first trimester assessment of this finding singly or in combination with other indices of uterine artery blood flow for early prediction of obstetric vasculopathies warranted a review. Rattanapuntamanee and Uerpairojkit determined uterine artery PI to describe the characteristic of uterine artery Doppler velocimetry in pregnant Thai subjects at 11 to $13(+6)$ gestational weeks. They found that when segregated for gestation age, uterine artery PI was $1.78 \pm 0.41$ at 11 weeks, $1.72 \pm 0.41$ at 
12 weeks, and $1.66 \pm 0.43$ at 13 weeks' gestation. The uterine artery $\mathrm{Pl}$ was $1.84 \pm 0.37$ with presence of notching and was $1.59 \pm 0.43$ without notching. The uterine artery notching was present in 19.3, 16 and $18 \%$ of the patients at 11, 12 and 13 weeks respectively. The uterine artery notching was absent in 18, 15.3 and $13.3 \%$ of patients at 11,12 and 13 weeks respectively. ${ }^{8}$ This group found that even in first trimester uterine artery diastolic notch in combination with other parameters seems to be a good test for prediction of obstetric vasculopathies particularly $\mathrm{PIH}$ and IUGR.

Another study assessed the role of uterine artery Doppler studies at 11 to 14 weeks in screening for pre-eclampsia (PET), small for gestational age (SGA) fetuses, and placental abruption-all major constitutes of obstetric vasculopathies. It is a prospective study on 1,123 subjects presenting for routine ultrasound examination at 11 to 14 weeks for nuchal translucency measurement. Uterine artery blood flow was studied by transvaginal color Doppler, the mean PI was calculated, and the presence of a diastolic notch was recorded. It was found that abnormal uterine Dopplers at 11 to 14 weeks identified one-third of subjects with severe early onset pre-eclampsia, all fetuses with SGA \pm th centile that were delivered at \pm 34 weeks, and $40 \%$ of cases with placental abruption. Uterine artery Doppler examination at the 11 to 14 weeks scan can identify a high risk population in which preventive or therapeutic interventions might be effective. $^{10}$

Another study undertaken to evaluate the association between uterine artery Doppler velocimetry performed between 10 and 14 weeks gestation and intrauterine growth restriction. The uterine artery mean RI for the entire cohort was equal on the right and left sides $(0.59 \pm 0.14)$. Of the 1067 subjects, 34.2\% had unilateral or bilateral diastolic notches, one notch was observed in $23.8 \%$, and bilateral notches in $10.4 \%$. It was found that subjects with a high uterine artery mean RI ( \pm 75th percentile) were 5.5 times more likely to have IUGR (95\% CI 1.6-18.7). There was no significant relationship between notching and IUGR. It concluded that elevated first trimester uterine artery mean RI is significantly associated with IUGR. ${ }^{11}$ This study further confirmed the efficacy of uterine artery diastolic notch in combination with other parameters in prediction of obstetric vasculopathies even in first trimester.

It therefore seems clear that even first trimester screening of uterine artery blood flow is reasonably competent in identifying high-risk subjects for developing conditions of obstetric vasculopathies. The advantage of such an early screening is obvious. It will allow an early instituting of preventive measures.

\section{DIASTOLIC NOTCH CONFIGURATION}

While presence or absence of diastolic notch is largely focused on in studying for predicting obstetric vasculopathies, it may be too simplistic to use only this aspect of the diastolic notch in prediction. Understandably therefore there are some recent studies that have started studying other features of diastolic notch as well.

\section{Notch Depth Index (NDI)}

One more concept that is being discussed currently as regards diastolic notch in predicting of obstetric vasculopathies and that is NDI. It is calculated as the depth of the diastolic notch divided by the maximal diastolic velocity (Fig. 4).

Ohkuchi A et al developed the NDI, to evaluate its association with the risk of pre-eclampsia and a small-forgestational-age (SGA) infant and to compare its clinical usefulness with that of the uterine artery RI and the peak systolic to early diastolic velocity (S/D) ratio. They studied in 288 consecutive healthy pregnant subjects at

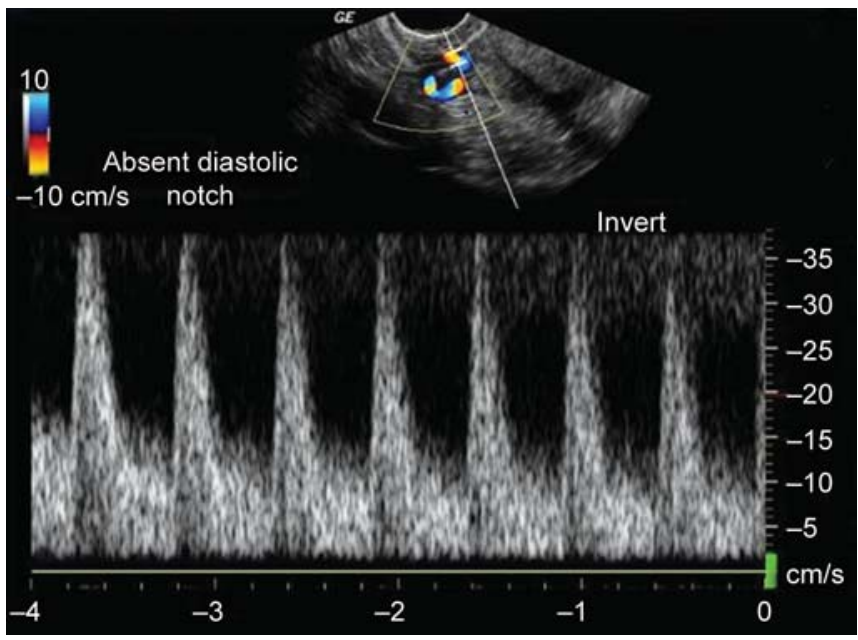

Fig. 3: Diastolic notch disappeared in first trimester itself

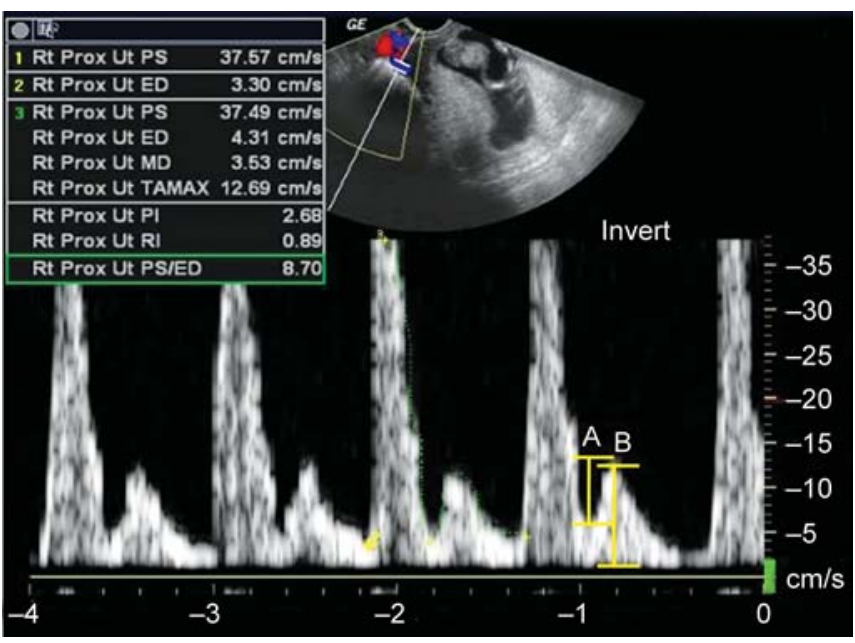

Fig. 4: Notch depth index 
$20.2 \pm 2.0$ (range 16.0-23.9) weeks of gestation and found that the NDI value in the second trimester is associated with the later onset of pre-eclampsia, and is clinically more useful in predicting pre-eclampsia than the two conventional indices. $^{12}$

\section{DIASTOLIC NOTCH IN COMBINATION WITH OTHER INDICES}

Many interesting studies have emerged on this aspect and all nearly consistently indicate to the fact that when uterine artery notching is combined with other indices the competence in predicting obstetric vasculopathies is increased. In one study relevant studies were identified through searches of MEDLINE, EMBASE, the Cochrane Library and Medion databases (all records to April 2006) and by checking bibliographies of identified studies and consulting with experts. Among 74 studies of pre-eclampsia (total 79,547 patients) and 61 studies of intrauterine growth restriction (total 41,131 patients) were identified. Uterine artery Doppler ultrasonography provided a more accurate prediction when performed in the second trimester than in the first-trimester. Most Doppler indices alone had poor predictive characteristics, but this varied with patient risk and outcome severity. An increased PI with notching was the best predictor of pre-eclampsia. It was also the best predictor of overall and severe intrauterine growth restriction among low-risk patients. The study concluded that abnormal uterine artery waveforms are a better predictor of preeclampsia than of intrauterine growth restriction. Pulsatility index, alone or combined with notching, is the most predictive Doppler index. ${ }^{13}$

In another study the scope of this study was further extended to third trimester as well. The aim of this study was to evaluate the different indices in the prediction of adverse outcome of pregnancies suspected for IUGR. Uterine artery blood flow was recorded in 217 pregnancies admitted for Doppler ultrasound surveillance due to suspected IUGR. The median gestational age at examination was 38 weeks (range 25-42 weeks). Only cases having bilateral uterine artery notching were included in the evaluation. The uterine artery Doppler spectrum was analyzed for different indices, including evaluation of notch and end-diastolic velocities. Umbilical artery Doppler velocimetry was also performed. The outcome variables chosen were: A small-for-gestational-age (SGA) newborn, preterm birth and abdominal delivery. It was found that the uterine artery blood velocity PI and RI were the best predictors of adverse outcome of pregnancy. ${ }^{14}$

In another study of a total of 614 primiparous subjects color flow/pulsed Doppler (CFPD) imaging of both uterine arteries at 20 weeks gestation was done. A receiver operator characteristic (ROC) curves were created for the systolic/ end-diastolic (A/B) ratio, RI and systolic/early diastolic (A/C) ratio for placental and nonplacental uterine arteries, individually or in combination with the presence of unilateral or bilateral notches. Based on data from ROC curves, the sensitivity of each method was compared with the false-positive rate set at 17 and $11 \%$. It was found that at 20 weeks' gestation, bilateral notches with mean RI cutoffs is the best screening method if further screening later in pregnancy is proposed. The $\mathrm{A} / \mathrm{C}$ ratio was complementary to bilateral notches when the false-positive rate was set at $17 \%$ and an effective quantitative substitute when the falsepositive rate was set at $11 \%{ }^{15}$

Reduced amniotic fluid clinically named as oligohydramnios is one more known manifestation of obstetric vasculopathies. It is an accompanying manifestation os preeclampsia and or IUGR. In one study abnormal Doppler velocimetry is related to adverse perinatal outcome for borderline amniotic fluid index during third trimester was studied. It was found that in the borderline AFI group, the presence of abnormal Doppler velocimetry measurement was related to increased risk of adverse perinatal outcome. The study concludes that borderline AFI of 5 to $8 \mathrm{~cm}$, especially when associated with abnormal Doppler velocimetry, mandates closer antenatal surveillance. ${ }^{16}$

\section{DIASTOLIC NOTCH AND OTHER RESISTANCE INDICES WITH CHEMICAL MARKERS}

While it is obvious from the above paragraphs that diastolic notch when combined with other color Doppler indices will increase its efficiency in predicting obstetric vasculopathies it would be of interest to review if combining diastolic notch studies with other markers can help improve its efficacy and reduce false positive rates.

One study concluded that while uterine artery Doppler screening identifies subjects at high risk for developing adverse pregnancy outcomes, detection rates may be increased and false positive rates reduced by combination with maternal characteristics or serum markers. ${ }^{17}$ Pedrosa and Matias performed a systematic review of screening for pre-eclampsia with the combination of uterine artery Doppler, maternal history, mean arterial pressure and/or maternal serum markers. They concluded that several tests provided moderate or convincing prediction of early PE, but screening for late pre-eclampsia was poor. Although, uterine artery Doppler is more accurate in the second trimester, they found encouraging results for first-trimester screening when it was combined with other markers. ${ }^{18}$

In one study the relationship between unexplained elevated serum markers in triple test, uterine artery Doppler measurements and adverse pregnancy outcome. It was found 
that patients with high AFP levels and bilateral uterine artery diastolic notch presence are candidates for pregnancy complications like low birthweight, preterm delivery and PROM. This study therefore felt that these groups of patients should be followed up more intensively. ${ }^{19}$

In another study longitudinal trend in fetoplacental biochemical markers, uterine artery PI and maternal blood pressure during the first trimester of pregnancy were studied. 86 subjects with singleton pregnancies were studied. In each woman, a blood sample was collected at 6 to 7,8 to 9 , 10 to 11 and 12 to 13 weeks' gestation. At the same visit blood pressure was measured and ultrasound examination was performed to measure the crown-rump length and Doppler flow velocity waveform patterns of both uterine arteries. Serum concentrations of pregnancy-associated plasma protein-A (PAPP-A), free $\beta$-human chorionic gonadotropin ( $\beta$-hCG), A disintegrin and metalloprotease domain-containing protein-12 (ADAM-12), placental protein-13 (PP-13) and placental growth factor (PlGF) levels were measured. It was found that there is a relationship between biochemical markers of early placentation and downstream resistance to flow in the uterine arteries in lowrisk uncomplicated pregnancies, indicating differences in placentation. In a small series of small for gestational age infants born at term this study could demonstrate differences as compared to normal pregnancies, with potential value for screening. ${ }^{20}$

In this regard a study by Gagnon A et al needs a good look at. This group has studied various obstetrical complications associated with abnormal maternal serum markers analytes. The Cochrane Library and Medline were searched for articles published from 1966 to February 2007, relating to maternal serum markers and perinatal outcomes. They found that abnormal maternal uterine artery Doppler in association with elevated maternal serum AFP, HCG, Inhibin A or decreased PAPP-A identifies a group of subjects at greater risk of IUGR and gestational hypertension with proteinuria. They therefore recommended that uterine artery Doppler measurements may be used in the evaluation of an unexplained abnormal level of either of these markers. ${ }^{21}$

It therefore seems clear from these results that combining uterine artery Doppler studies with other markers increases its efficiency in predicting obstetric vasculopathies.

\section{EFFECT OF SOME TREATMENT METHODS ON DIASTOLIC NOTCH AND OTHER RESISTANCE INDICES}

While this review firmly establishes an important role of uterine color Doppler in prediction of obstetric vasculopathies, it will be of relevance to review if some treatment modalities have any effect on them. In one study usefulness of aspirin therapy in high-risk pregnant subjects with abnormal uterine artery Doppler ultrasound at 14 to 16 weeks pregnancy was studied through a randomized controlled clinical trial. The trial enrolled 139 subjects at risk of preeclampsia or IUGR, with abnormal uterine artery Doppler findings that included the presence of unilateral or bilateral diastolic notch, high RI, or high PI at 14 to 16 weeks of gestation. The subjects were randomly allocated into two groups, one receiving aspirin since admission to hospital $(n=74)$ and the other serving as control $(n=65)$. All subjects were followed up until delivery to assess maternal and perinatal outcomes. It was found that low-dose aspirin administered as early as 14 to 16 weeks of gestation to pregnant subjects at high risk of pre-eclampsia with abnormal uterine Doppler findings may reduce or modify the course of severe pre-eclampsia and may help in prevention of IUGR. ${ }^{22}$

In another study effect of magnesium sulfate on PI of uterine, umbilical and fetal middle cerebral arteries is according to the persistence of bilateral diastolic notch of uterine arteries in patients with severe pre-eclampsia. This is a cohort study including 40 pregnant subjects with severe pre-eclampsia, 23 of them presenting bilateral protodiastolic notch, and 17, unilateral/absent notch. These patients were submitted to Doppler velocimetry before and 20 minutes after the intravenous administration of $6 \mathrm{gm}$ of magnesium sulfate. The difference between the two measurements (before and after magnesium sulfate) was compared between the groups. It was found that after the intravenous administration of 6 gm of magnesium sulfate to patients with severe pre-eclampsia, a significant decrease was found in blood pressure and in the PI of the uterine, umbilical and fetal middle cerebral arteries. ${ }^{23}$

\section{REFERENCES}

1. Chan FY, Pun TC, Lam C, et al. Pregnancy screening by uterine artery doppler velocimetry-which criterion performs the best. Obstet Gynecol 1995;85:696-702.

2. Thangaratinam S, Langenveld J, Mol BW, Khan KS. Prediction and primary prevention of pre-eclampsia. Best Pract Res Clin Obstet Gynaecol. 2011 Aug;25(4):419-33.

3. Antsaklis A, Daskalakis G, Tzortzis E, et al. The effect of gestational age and placental location on the prediction of pre-eclampsia on uterine artery Doppler velocimetry in lowrisk nulliparous subjects. Ultrasound Obstet Gynecol 2000;16: 635-39.

4. Prefumo F, Güven M, Ganapathy R, Thilaganathan B. Longitudinal variation in uterine artery blood flow pattern in relation to birth weight. Obstet Gynecol 2004 Apr;103(4):764-68.

5. Pongrojpaw D, Chanthasenanont A, Nanthakomon T. Second trimester uterine artery Doppler screening in prediction of adverse pregnancy outcome in high risk subjects. J Med Assoc Thai 2010 Dec;93 (Suppl) 7:S127-30. 
6. Espinoza J, Kusanovic JP, Bahado-Singh R, Gervasi MT, Romero R, Lee W, et al. Should bilateral uterine artery notching be used in the risk assessment for pre-eclampsia, small-forgestational-age and gestational hypertension? J Ultrasound Med 2010 Jul;29(7):1103-15.

7. Park YW, Lim JC, Kim YH, Kwon HS. Uterine artery Doppler velocimetry during mid-second trimester to predict complications of pregnancy based on unilateral or bilateral abnormalities. Yonsei Med J 2005 Oct 31;46(5):652-57.

8. Rattanapuntamanee $\mathrm{O}$, Uerpairojkit B. Reference range and characteristic of uterine artery Doppler in pregnant Thai subjects at 11-13(+6) gestational weeks. J Med Assoc Thai 2011 Jun; 94(6):644-48.

9. Geipel A, Hennemann F, Fimmers R, Willruth A, Lato K, Gembruch U, Berg C. Reference ranges for Doppler assessment of uterine artery resistance and pulsatility indices in dichorionic twin pregnancies. Ultrasound Obstet Gynecol 2011 Jun; 37(6): 663-67.

10. Pilalis A, Souka AP, Antsaklis P, Basayiannis K, Benardis P, Haidopoulos D, et al. Screening for pre-eclampsia and small for gestational age fetuses at the 11-14 weeks scan by uterine artery Dopplers. Acta Obstet Gynecol Scand 2007;86(5):530-34.

11. Dugoff L, Lynch AM, Cioffi-Ragan D, Hobbins JC, Schultz LK, Malone FD, et al. FASTER Trial Research Consortium. First trimester uterine artery Doppler abnormalities predict subsequent intrauterine growth restriction. Am J Obstet Gynecol 2005 Sep;193(3 Pt 2):1208-12.

12. Ohkuchi A, Minakami H, Sato I, Mori H, Nakano T, Tateno M. Predicting the risk of pre-eclampsia and a small-for-gestationalage infant by quantitative assessment of the diastolic notch in uterine artery flow velocity waveforms in unselected subjects. Ultrasound Obstet Gynecol 2000 Aug;16(2):171-78.

13. Cnossen JS, Morris RK, ter Riet G, Mol BW, van der Post JA, Coomarasamy A, et al. Use of uterine artery Doppler ultrasonography to predict pre-eclampsia and intrauterine growth restriction: A systematic review and bivariable meta-analysis. CMAJ 2008 Mar 11;178(6):701-11.

14. Ghosh G, Breborowicz A, Brazert M, Maczkiewicz M, Kobelski M, Dubiel M, et al. Evaluation of third trimester uterine artery flow velocity indices in relationship to perinatal complications. J Matern Fetal Neonatal Med 2006 Sep; 19(9):551-55.

15. Aquilina J, Barnett A, Thompson O, Harrington K. Comprehensive analysis of uterine artery flow velocity waveforms for the prediction of pre-eclampsia. Ultrasound Obstet Gynecol 2000 Aug;16(2):163-70.
16. Kwon JY, Kwon HS, Kim YH, Park YW. Abnormal Doppler velocimetry is related to adverse perinatal outcome for borderline amniotic fluid index during third trimester. J Obstet Gynaecol Res 2006 Dec;32(6):545-49.

17. Papageorghiou AT, Leslie K. Uterine artery Doppler in the prediction of adverse pregnancy outcome. Curr Opin Obstet Gynecol 2007 Apr;19(2):103-09.

18. Pedrosa AC, Matias A. Screening for pre-eclampsia: A systematic review of tests combining uterine artery Doppler with other markers. J Perinat Med 2011 Nov;39(6):619-35.

19. Karsidag AY, Buyukbayrak EE, Kars B, Suyugul U, Unal O, Turan MC. The relationship between unexplained elevated serum markers in triple test, uterine artery Doppler measurements and adverse pregnancy outcome. J Pak Med Assoc 2010 Mar; 60(3):181-86.

20. Wortelboer EJ, Koster MP, Kuc S, Eijkemans MJ, Bilardo CM, Schielen PC, et al. Longitudinal trends in fetoplacental biochemical markers, uterine artery pulsatility index and maternal blood pressure during the first trimester of pregnancy. Ultrasound Obstet Gynecol 2011 Oct;38(4):383-88.

21. Gagnon A, Wilson RD, Audibert F, Allen VM, Blight C, Brock JA, et al. Society of obstetricians and gynaecologists of Canada Genetics Committee. Obstetrical complications associated with abnormal maternal serum markers analytes. J Obstet Gynaecol Can 2008 Oct;30(10):918-49.

22. Ebrashy A, Ibrahim M, Marzook A, Yousef D. Usefulness of aspirin therapy in high-risk pregnant subjects with abnormal uterine artery Doppler ultrasound at 14-16 weeks pregnancy: Randomized controlled clinical trial. Croat Med J 2005 Oct; 46(5):826-31.

23. Souza AS, Amorim MM, Santos RE, Noronha Neto C, Porto A. Effect of magnesium sulfate on pulsatility index of uterine, umbilical and fetal middle cerebral arteries according to the persistence of bilateral diastolic notch of uterine arteries in patients with severe pre-eclampsia: Rev Bras Ginecol Obstet 2009 Feb;31(2):82-88.

\section{ABOUT THE AUTHOR}

\section{Pankaj Desai}

Chief, Department of Obstetrics and Gynecology (VRS), Medical College and SSG Hospital, Vadodara, Gujarat, India, Phone: 91-265-2437793, 2432519, e-mail: drpankajdesai@gmail.com 